\title{
SINAIS E SINTOMAS RELACIONADOS À INALAÇÃO DA FUMAÇA CIRÚRGICA POR CIRURGIÕES
}

\author{
Signs and symptoms related to inhalation of surgical smoke by surgeons
}

\author{
Signos y sintomas relacionados con la inhalación del humo quirúrgico por cirujanos \\ Jéssica Ribeiro Aranha ${ }^{1 *}$ (1), Renata Perfeito Ribeiro² (D), Amanda Salles Margatho² (D),

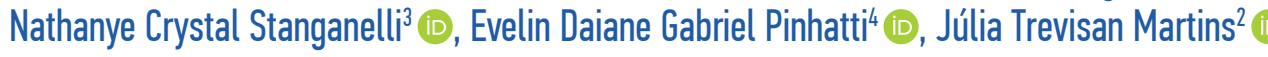

RESUMO: Objetivo: Verificar a associação entre a prevalência de sinais e sintomas relacionados à inalação da fumaça cirúrgica e o tempo de atuação desde a formatura dos cirurgiões expostos. Método: Estudo de campo, transversal, descritivo, quantitativo. Os dados foram coletados por uma das pesquisadoras em entrevista individual com 45 cirurgiões, com o auxílio de um instrumento contendo dados sociodemográficos e variáveis referentes aos sinais e sintomas relacionados à inalação da fumaça cirúrgica descritos na literatura. Resultados: A amostra foi composta, em sua maioria, de cirurgiões do sexo masculino, com prevalência da clínica de ginecologia e obstetrícia. Não houve significância estatística entre a associação dos sinais e sintomas relacionados à inalação da fumaça e o tempo de atuação desde a formatura dos cirurgiões expostos ( $\mathrm{p}>0,05)$. Conclusão: Houve maior prevalência dos sintomas irritação nos olhos e sensação de corpo estranho na garganta entre os cirurgiões com mais de 30 anos na função desde a formatura; $60,0 \%$ dos cirurgiões não acreditam que os sintomas estejam relacionados à inalação da fumaça cirúrgica. Recomenda-se, para a minimização dos sinais e sintomas, a instalação de exaustores de fumaça em salas cirúrgicas e o uso da máscara N95 pelos trabalhadores expostos a esse risco.

Palavras-chave: Cirurgiões. Sinais e sintomas. Eletrocoagulação. Saúde do trabalhador.

ABSTRACT: Objective: To determine the association between the prevalence of signs and symptoms related to the inhalation of surgical smoke and time in practice of the exposed surgeons. Method: A cross-sectional, descriptive, quantitative field study was conducted. The data were collected by one of the authors in an individual interview with 45 surgeons, with the aid of an instrument aimed at collecting sociodemographic information and data on variables referring to signs and symptoms related to the inhalation of surgical smoke described in the literature. Results: The sample consisted mostly of male surgeons, mostly from the obstetrics and gynecology clinic. There was no statistical significance between the association of signs and symptoms related to smoke inhalation and time in practice of the exposed surgeons ( $\mathrm{p}>0.05)$. Conclusion: There was a higher prevalence of eye irritation symptoms and foreign-body sensation in the throat in surgeons with more than 30 years of practice; $60.0 \%$ of surgeons did not believe that the symptoms were related to the inhalation of surgical smoke. It is recommended that an exhaust fan be installed in the operating room and that an N95 mask be used by workers exposed to this risk to minimize the signs and symptoms of smoke inhalation.

Key words: Surgeons. Signs and symptoms. Electrocoagulation. Occupational health.

RESUMEN: Objetivo: Determinar la asociación entre la prevalencia de signos y síntomas relacionados con la inhalación de humo quirúrgico y el tiempo en la práctica de los cirujanos expuestos. Método: Se realizó un estudio de campo cuantitativo, descriptivo y de corte transversal. Los datos fueron recopilados por uno de los autores en una entrevista individual con 45 cirujanos, con la ayuda de un instrumento destinado a recopilar información sociodemográfica y datos sobre variables relacionadas con signos y síntomas relacionados con la inhalación de humo quirúrgico descrita en la literatura. Resultados: la muestra estuvo compuesta principalmente por cirujanos varones, principalmente de la clínica de obstetricia y ginecología. No hubo significación estadística entre la asociación

\footnotetext{
'Enfermeira; residente em Enfermagem Perioperatória do Departamento de Enfermagem da Universidade Estadual de Londrina (UEL) - Londrina (PR), Brasil. ${ }^{2}$ Enfermeira; professora doutora adjunta do Departamento de Enfermagem da UEL - Londrina (PR), Brasil.

${ }^{3}$ Enfermeira; mestranda do Programa de Pós-Graduação em Enfermagem do Departamento de Enfermagem da UEL - Londrina (PR), Brasil.

“Enfermeira; doutoranda do Programa de Pós-Graduação em Enfermagem do Departamento de Enfermagem da UEL - Londrina (PR), Brasil.

*Autor correspondente: jessica_aranha10@hotmail.com

Recebido: 27/03/2018 - Aprovado: 28/08/2018

DOI: $10.5327 / Z 1414-4425201800040005$
} 
de signos y síntomas relacionados con la inhalación de humo y el tiempo en la práctica de los cirujanos expuestos (p $>0.05$ ). Conclusión: Hubo una mayor prevalencia de síntomas de irritación ocular y sensación de cuerpo extraño en la garganta en cirujanos con más de 30 años de práctica; El $60,0 \%$ de los cirujanos no creía que los síntomas estuvieran relacionados con la inhalación de humo quirúrgico. Se recomienda que se instale un ventilador de extracción en la sala de operaciones y que los trabajadores expuestos a este riesgo utilicen una máscara N95 para minimizar los signos y síntomas de la inhalación de humo. Palabras clave: Cirujanos. Signos y síntomas. Electrocoagulación. Salud laboral.

\section{INTRODUÇÃ̃O}

O Centro Cirúrgico (CC) pode ser considerado uma das unidades mais complexas de uma instituição hospitalar, considerando-se suas especificidades e o constante risco à saúde tanto para o paciente, em relação à intervenção cirúrgica necessária, quanto para os trabalhadores, devido aos procedimentos realizados durante a prestação da assistência ${ }^{1}$.

O eletrocautério é uma das tecnologias baseada em radiofrequência utilizada constantemente no CC em diferentes especialidades, de maneira que facilite a visualização do campo operatório e diminua sangramentos e tempo cirúrgico ${ }^{1}$.

Esse equipamento pode ser monopolar ou bipolar. O monopolar, por sua versatilidade e eficiência, é mais utilizado na dissecção e coagulação de vasos. Já a cauterização bipolar é empregada em tecidos delicados que se encontram entre os eletrodos². Não há diferença entre o eletrocautério monopolar e o bipolar em relação à concentração dos compostos químicos gerados durante $o$ seu uso e liberados no ambiente em forma de fumaça cirúrgica ${ }^{3}$.

A fumaça cirúrgica é formada pela cauterização incompleta dos tecidos e apresenta, em sua composição, gases tóxicos que podem se acumular em forma de material orgânico vivo ou morto, sendo prejudicial à saúde daqueles que a inalam ${ }^{1}$, como é o caso de cirurgiões, anestesistas e equipe de enfermagem do CC.

Essa fumaça pode conter vírus, bactérias e contaminantes químicos e biológicos - 95\% dela é composta de água, e os outros $5 \%$, de partículas potencialmente prejudiciais à saúde ${ }^{4,5}$. Os gases tóxicos de odor fétido, formados a partir da fumaça cirúrgica, liberam pequenas partículas que podem provocar complicações respiratórias e agentes patogênicos que podem ser transmitidos para a equipe cirúrgica ${ }^{6,7}$.

As substâncias produzidas pela fumaça cirúrgica podem ser absorvidas pela pele ou via respiratória dos cirurgiões expostos durante suas atividades laborais, na forma de poeiras, fumaça, névoa, gases ou vapores, sendo essa fumaça um dos principais riscos químicos presentes nas salas operatórias $(\mathrm{SO})^{8}$.

O risco químico da fumaça cirúrgica está relacionado ao odor, ao tamanho das partículas e à concentração dos gases. A grande ameaça está representada pelas toxinas que causam o odor, as quais são liberadas no ar quando o tecido é cauterizado pelo eletrocautério. Esse odor é característico de produtos químicos formados a partir da combustão de proteínas e lipídeos no ato do uso do eletrocautério ${ }^{4,9-12}$. Esses produtos químicos causam cefaleia, sensação de corpo estranho na garganta, náuseas, vômitos, irritação dos olhos, fraqueza, tontura ${ }^{13}$, ardência na faringe, congestão nasal, espirros e irritação da mucosa nasal e cavidade oral ${ }^{1}$.

A fumaça cirúrgica possui como substâncias químicas hidrocarbonetos policíclicos aromáticos (HPA $)^{14,15}$, compostos orgânicos voláteis $(\mathrm{COV})^{16}$, monóxido de carbono $(\mathrm{CO})^{3}$, tolueno ${ }^{17}$, entre outras. Esses compostos químicos são responsáveis por características mutagênicas ${ }^{18} \mathrm{e} /$ ou cancerígenas ${ }^{19}$, dependendo do tempo de exposição do trabalhador.

Em estudo com o objetivo de comparar os riscos relacionados à inalação de fumaça cirúrgica em procedimentos laparoscópicos com procedimentos abertos, constatou-se que, em cirurgias laparoscópicas, a fumaça cirúrgica permanece na cavidade abdominal do paciente até a abertura da válvula do trocarte para que seja liberada. A análise dessa fumaça revelou a presença de substâncias carcinogênicas como etanol, dicloroetano, benzeno e etilbenzeno ${ }^{16}$.

A literatura nacional e a internacional não apresentam estudos que comprovem a relação entre a exposição à fumaça cirúrgica e a presença de sinais e sintomas em cirurgiões expostos. Tendo isso em vista, tem-se a seguinte pergunta de pesquisa: existe associação entre a prevalência de sinais e sintomas relacionados à inalação da fumaça cirúrgica e o tempo de atuação desde a formatura dos cirurgiões expostos?

\section{OBJETIVO}

Verificar a associação entre a prevalência de sinais e sintomas relacionados à inalação de fumaça cirúrgica e o tempo de atuação desde a formatura dos cirurgiões expostos.

\section{MÉTODO}

Trata-se de um estudo de campo, transversal, descritivo, quantitativo, realizado no período de fevereiro a junho de 2016. 
A população de estudo foi constituída de cirurgiões de diversas clínicas em uma cidade no norte do Paraná, que atendiam determinado convênio médico privado do município. Para a amostra, foram estabelecidos os seguintes critérios de inclusão: ser cirurgião e estar exposto à inalação da fumaça cirúrgica produzida pelo eletrocautério pelo menos uma vez na semana. Como critério de exclusão, estabeleceu-se ser tabagista, pois este pode apresentar sintomas semelhantes aos produzidos pela exposição à fumaça cirúrgica.

Os cirurgiões foram selecionados com base em informações do convênio de saúde que atendiam. Os dados foram coletados por uma das pesquisadoras, em entrevista individual, no consultório do cirurgião, após agendamento de horário com sua secretária.

O instrumento de coleta de dados utilizado foi composto de variáveis sociodemográficas - sexo, idade, clínica e há quantos anos exercia a profissão de cirurgião - e variáveis clínicas, referentes à presença de sinais e sintomas relacionados à inalação da fumaça cirúrgica - cefaleia, sensação de corpo estranho na garganta, náuseas, vômitos, irritação nos olhos, fraqueza, tontura ${ }^{13}$, ardência na faringe, congestão nasal, irritação da mucosa nasal e cavidade oral e espirros ${ }^{1}$. Ao fim da entrevista, questionou-se aos cirurgiões se consideravam que a presença desses sinais e sintomas poderia estar relacionada à inalação da fumaça cirúrgica.

Para a análise estatística, foi utilizado o software Statistical Package for the Social Sciences (SPSS), versão 20.0 para Windows. Foram realizadas análises descritivas de frequência simples para as variáveis categóricas, de tendência central (média) e de dispersão (desvio padrão, valores mínimo e máximo) para as variáveis numéricas. Para verificar as possíveis associações entre os sinais e sintomas relacionados à inalação da fumaça cirúrgica e o tempo após formação do cirurgião, foi realizado o teste exato de Fisher. O nível de significância adotado foi de 0,05.

O projeto foi aprovado pelo Comitê de Ética em Pesquisa, via Plataforma Brasil, sob CAAE $n^{\circ}$ 46229915.0.0000.5231, segundo a Resolução $n^{\circ}$ 466 / 2012, do Conselho Nacional de Saúde. Os cirurgiões foram orientados sobre o estudo e, ao aceitarem participar, assinaram o Termo de Consentimento Livre e Esclarecido (TCLE).

\section{RESULTADOS}

A amostra deste estudo foi constituída de 45 cirurgiões. Na Tabela 1, apresenta-se a caracterização sociodemográfica deles.

$\mathrm{Na}$ Tabela 2, exibe-se a associação entre a prevalência de sinais e sintomas relacionados à inalação da fumaça cirúrgica e o tempo de atuação desde a formatura dos cirurgiões expostos.
Na Tabela 3, expressa-se a prevalência dos sinais e sintomas relacionados à inalação da fumaça do eletrocautério.

Quando questionados se a presença dos sinais e sintomas estava relacionada à inalação da fumaça cirúrgica, $60 \%$ $(n=27)$ dos entrevistados responderam que não e $40 \%(n=18)$ acreditavam que havia relação.

\section{DISCUSSÃO}

De acordo com uma pesquisa realizada no México, $86 \%$ dos entrevistados eram do sexo masculino, assim como nesta pesquisa. No entanto, os sintomas mais frequentes apresentaram prevalência diferente do que se obteve neste estudo, pois, naquele, a sensação de corpo estranho na garganta representou $58 \%$; ardência na faringe, $22 \%$; náuseas, $4 \%$; e congestão nasal, $2 \%$. As especialidades com maior índice de exposição ao eletrocautério foram neurocirurgia, cirurgia geral e ginecologia e obstetrícia ${ }^{1}$.

No ambiente de trabalho dos cirurgiões, a fumaça cirúrgica contendo gases e partículas químicas de diversos tamanhos é

Tabela 1. Caracterização sociodemográfica dos cirurgiões expostos à inalação da fumaça cirúrgica.

\begin{tabular}{|l|c|c|}
\hline Variáveis & $\begin{array}{c}\text { Até } \mathbf{3 0} \text { anos de } \\
\text { formado (n=21) }\end{array}$ & $\begin{array}{c}\text { Mais de } \mathbf{3 0} \text { anos de } \\
\text { formado (n=24) }\end{array}$ \\
\hline $\begin{array}{l}\text { Idade em anos } \\
\text { média (DP) }\end{array}$ & $51(4,0)$ & $\mathbf{n}(\mathbf{\%})$ \\
\hline Mínimo & 43 & $54(6,0)$ \\
\hline Máximo & 59 & 79 \\
\hline Sexo & & \\
\hline Masculino & $16(46,7)$ & $22(48,9)$ \\
\hline Feminino & $05(11,1)$ & $02(4,4)$ \\
\hline Clínica & & \\
\hline Ginecologia & $12(26,7)$ & $09(20,1)$ \\
\hline Obstetrícia & $05(11,1)$ & $04(8,9)$ \\
\hline Urologia & $00(0,0)$ & $04(8,9)$ \\
\hline Vascular & $02(4,4)$ & $01(2,2)$ \\
\hline Torácica & $00(0,0)$ & $03(6,7)$ \\
\hline Neurologia & $00(0,0)$ & $02(4,4)$ \\
\hline Cardíaca & $01(2,2)$ & $00(0,0)$ \\
\hline Ortopedia & $01(2,2)$ & $00(0,0)$ \\
\hline Proctologia & $00(0,0)$ & $01(2,2)$ \\
\hline Pediátrica & & \\
\hline
\end{tabular}

DP: desvio padrão. 
motivo de preocupações em relação à saúde desses trabalhadores, pois a maioria dos sintomas ocorre, na prática, em longo prazo $^{6,10}$. Embora não tenha apresentado significância estatística, observou-se maior prevalência dos sintomas irritação dos olhos e sensação de corpo estranho na garganta (Tabela 2) entre os cirurgiões com mais de 30 anos de atuação desde a formatura.

Por sua vez, a fumaça do eletrocautério apresenta uma série de riscos para a saúde, entre os quais se cita:

- Inalação: $90 \%$ das partículas presentes na fumaça cirúrgica são tão pequenas que podem penetrar no

Tabela 2. Associação entre a prevalência de sinais e sintomas relacionados à inalação da fumaça cirúrgica e o tempo após formação do cirurgião.

\begin{tabular}{c|c|c|c|}
\multirow{2}{*}{ Variáveis } & $\begin{array}{c}\text { Até } 30 \text { anos de } \\
\text { formado }(n=21)\end{array}$ & $\begin{array}{c}\text { Mais de } 30 \text { anos } \\
\text { de formado }(n=24)\end{array}$ & $\begin{array}{c}\text { Valor } \\
\text { P* }\end{array}$ \\
\cline { 2 - 3 } & $n(\%)$ & $n(\%)$ & \\
\hline
\end{tabular}

Irritação nos olhos

\begin{tabular}{c|c|c|c|}
\hline Sim & $04(8,9)$ & $04(8,9)$ & 0,83 \\
\hline Não & $17(37,8)$ & $20(44,4)$ & \\
\hline
\end{tabular}

Espirros

\begin{tabular}{|c|c|c|c|}
\hline Sim & $03(6,7)$ & $01(2,2)$ & 0,23 \\
\hline Não & $18(40,0)$ & $23(51,1)$ & \\
\hline
\end{tabular}

Sensação de corpo estranho na garganta

\begin{tabular}{c|c|c|c|} 
Sim & $01(2,2)$ & $02(4,4)$ & 0,63 \\
\hline Não & $20(44,4)$ & $22(48,9)$ & \\
\hline
\end{tabular}

\section{Ardência na faringe}

\begin{tabular}{c|c|c|c|} 
Sim & $01(2,2)$ & $01(2,2)$ & 0,92 \\
\hline Não & $20(44,4)$ & $23(51,1)$ & \\
\hline
\end{tabular}

\begin{tabular}{|c|c|c|}
\hline \multicolumn{2}{|c|}{ Congestão nasal } \\
\hline Sim & $02(4,4)$ & $00(0,0)$ \\
\hline Não & $19(42,2)$ & $24(53,3)$ \\
\hline
\end{tabular}

\begin{tabular}{|c|c|c|c|}
\hline \multicolumn{4}{|c|}{ Fraqueza } \\
\hline Sim & $00(0,0)$ & $01(2,2)$ & \multirow{2}{*}{0,34} \\
\hline Não & $21(46,7)$ & $23(51,1)$ & \\
\hline \multicolumn{4}{|c|}{ Cefaleia } \\
\hline Sim & $00(0,0)$ & $01(2,2)$ & \multirow{2}{*}{0,34} \\
\hline Não & $21(46,7)$ & $23(51,1)$ & \\
\hline \multicolumn{4}{|c|}{ Tontura } \\
\hline Sim & $00(0,0)$ & $01(2,2)$ & \multirow{2}{*}{0,34} \\
\hline Não & $21(46,7)$ & $23(51,1)$ & \\
\hline \multicolumn{4}{|c|}{ Náusea e/ou vômitos } \\
\hline Sim & $01(2,2)$ & $00(0,0)$ & \multirow{2}{*}{0,28} \\
\hline Não & $20(44,4)$ & $24(53,3)$ & \\
\hline
\end{tabular}

alvéolo do trabalhador. Essas partículas normalmente causam irritação no nariz e na garganta e problemas respiratórios, podendo também ocorrer reações alérgicas. Quanto menores essas partículas, mais perigosas são do ponto de vista químico ${ }^{20}$;

- Má qualidade do ar: durante o corte e a coagulação feitos com o eletrocautério, sente-se um odor desagradável no ambiente, o qual prejudica a qualidade do ar nas $\mathrm{SO}$, a ponto de a equipe sentir cefaleia e náuseas ${ }^{20}$;

- Visão prejudicada: a fumaça do eletrocautério pode dificultar a visão durante os procedimentos, por restringir a visão do campo operatório e também por irritar os olhos ${ }^{20}$;

- Máscaras cirúrgicas: ao contrário do que se acredita, essas máscaras convencionais não oferecem proteção suficiente contra a fumaça cirúrgica, pois não impedem a inalação dos gases tóxicos e aerossóis. Elas foram desenvolvidas para proteger o paciente de infecções durante os procedimentos cirúrgicos ${ }^{20} \mathrm{e}$ formam barreira para resguardar a face do cirurgião contra grandes gotas e respingos de sangue e outros fluidos corporais ${ }^{21}$, porém não fornecem uma proteção adequada contra a fumaça do eletrocautério ${ }^{13}$.

A opção seria o uso de máscaras de filtro com qualidade superior ou máscaras duplas, que poderiam aumentar a capacidade de filtração, e um dispositivo de extração da fumaça posicionado de 2 a $5 \mathrm{~cm}$ do local de uso do eletrocautério, garantindo, assim, a segurança dos cirurgiões ${ }^{13}$. Ou, então, máscara N-95, que impede a passagem do material particulado atmosférico considerado perigoso e, portanto, é apontada como um ótimo método para a prevenção dos sinais e sintomas relacionados ao uso do eletrocautério ${ }^{22}$.

Tabela 3. Prevalência dos sinais e sintomas relacionados à inalação da fumaça do eletrocautério.

\begin{tabular}{|l|c|}
\hline Sinais e sintomas & $\mathbf{n}(\%)$ \\
\hline Irritação nos olhos & $08(17,8)$ \\
\hline Espirros & $04(8,9)$ \\
\hline Sensação de corpo estranho na garganta & $03(6,6)$ \\
\hline Ardência na faringe & $02(4,4)$ \\
\hline Congestão nasal & $02(4,4)$ \\
\hline Fraqueza & $01(2,2)$ \\
\hline Cefaleia & $01(2,2)$ \\
\hline Tontura & $01(2,2)$ \\
\hline Náuseas e/ou vômitos & $01(2,2)$ \\
\hline Irritação da mucosa nasal e cavidade oral & $00(0,0)$ \\
\hline
\end{tabular}


Reconhece-se a fumaça cirúrgica como um produto químico potencialmente arriscado para a saúde dos trabalhadores expostos ${ }^{23}$. A solução para o gerenciamento dessa fumaça e de seus sintomas é a sua evacuação completa, para que o ar fique constantemente limpo, garantindo um ambiente seguro para um trabalho saudável ${ }^{24}$.

Portanto, capturar a fumaça cirúrgica é a capacidade de recolhê-la durante um procedimento cirúrgico e removê-la para uma área distante da equipe, onde ela possa ser filtrada. Um exemplo são os ventiladores de exaustão locais, recomendados pelas organizações profissionais e agências de saúde governamentais. Essas máquinas estão ligadas a filtros que têm carvão ativado, absorvendo produtos químicos e odores presentes na fumaça. Os filtros ultra-low penetration air (ULPA) removem 99,9\% de contaminantes que medem 0,12 mícrons ou mais em questão de diâmetro, filtrando o ar de grande quantidade de fumaça cirúrgica ${ }^{5,25}$.

As razões para que o aspirador de fumaça não seja utilizado têm relação com a falta de conhecimento sobre os riscos da inalação dessa fumaça, sendo, muitas vezes, o fator decisivo na escolha de utilizar ou não os dispositivos de aspiração. A recusa para realizar a aspiração da fumaça pode ser explicada por: ansiedade associada a qualquer alteração de rotinas; falta de conhecimento sobre as fontes que recomendam a remoção da fumaça; incômodo causado pelo ruído gerado pelo aspirador de fumaça; indisponibilidade de dispositivos que permitam atingir alta eficiência de captura da fumaça; dispositivos que exigem o envolvimento de toda a equipe cirúrgica ${ }^{26}$. Tais dados corroboram este estudo, que revela o descrédito por parte dos cirurgiões $(60,0 \%)$ sobre os riscos de apresentarem sinais e sintomas que envolvam o uso do eletrocautério.

Detalha-se como limitações deste estudo o número reduzido da amostra de cirurgiões que participaram, pois, muitas vezes, não apresentaram disponibilidade para atender um das pesquisadoras. O pequeno tamanho amostral pode ter relação com as associações não significativas entre a presença dos sinais e sintomas relacionados à inalação da fumaça cirúrgica e o tempo de atuação desde a formatura dos cirurgiões expostos.

Contudo, este estudo avança no conhecimento científico em relação às consequências negativas que podem afetar a saúde de trabalhadores sujeitos à inalação da fumaça cirúrgica. Conhecer as alterações que essa inalação pode causar nos cirurgiões é de grande importância para a implantação de ações que minimizem essa exposição.

\section{CONCLUSÃO}

Este estudo não encontrou significância estatística entre a associação dos sinais e sintomas e o tempo após formação entre os cirurgiões estudados. Contudo, observou-se maior prevalência dos sintomas irritação nos olhos e sensação de corpo estranho na garganta entre os cirurgiões com mais de 30 anos de atuação desde a formatura. Ressalta-se que $60,0 \%$ deles não acredita que os sintomas estejam relacionados à inalação da fumaça cirúrgica. Recomenda-se, para a minimização dos sinais e sintomas, a instalação de exaustores de fumaça em SO e o uso da máscara N95 pelos trabalhadores expostos ao risco.

Por fim, sugerem-se novos estudos em relação a essa máscara, no intuito de produzir evidência científica que justifique o uso desse equipamento de proteção individual para a retenção dos componentes químicos da fumaça cirúrgica, pois esta é considerada prejudicial ao trabalhador exposto a ela pela literatura. Além disso, estudos de acompanhamento para determinar o tempo de aparecimento dos sinais e sintomas e o desenvolvimento de escalas que possam mensurá-los também serão importantes.

\section{REFERÊNCIAS}

1. Navarro-Meza MC, González-Baltazar R, Aldrete-Rodríguez MG, Carmona-Navarro DH, López-Cardona MG. Síntomas respiratorios causados por el uso delelectrocauterio em médicos em formaciónquirúrgica de un hospital de México. Rev Peru Med Exp Salud Publica [Internet]. 2013 [acessado em 5 fev. 2018];30(1):41-4. Disponível em: http://dx.doi.org/10.1590/ S1726-46342013000100008

2. Cervantes-Sánchez CE, Cu-Zetina C, Seviano-Rico E, Rojero-Vallejo J, Lazos-Ochos M, Gutiérrez-Vega R. Incisión cutânea: bisturi vselectrocauterio. Estudio experímental em ratas. Rev Med Hosp Gen Mex. 2002;65(1):11-4.
3. Gianella M, Hahnloser D, Rey JM, Sigrist MW. Quantitative chemical analysis of surgical smoke generated during laparoscopic surgery with a vessel-sealing device. Surg Innov. 2014;21(2):170-9. https:// doi.org/10.1177/1553350613492025

4. Ulmer BC. The hazards of surgical smoke. AORN J. 2008;87(4):721-34.

5. National Institute for Occupational Safety and Health. Control of smoke from laser/electric surgical procedures [Internet]. Estados Unidos: National Institute for Occupational Safety and Health; 1996 [acessado em 15 set. 2017];96-128. Disponivel em: https://www. cdc.gov/niosh/docs/hazardcontrol/pdfs/hc11.pdf 
6. Hensman C, Baty D, Willis RG, Cuschieri A. Chemical compositions of smoke producedby high-frequency electrosurgery in a closed gaseous environment: an in vitro study. Surg Endosc. 1998;12(8):1017-9.

7. Moot AR, Ledingham KM, Wilson PF, SenthilmohanST, Lewis DR, Roake $\mathrm{J}$, et al. Composition of volatile organic compounds in diathermy plume as detected by selected ion flow tube mass spectrometry. ANZ J Surg. 2007;77(1-2):20-3. http://dx.doi.org/10.1111/j.1445-2197.2006.03827.x

8. Scaldelai VS, Oliveira CAD, Milaneli E, Oliveira JBC, Bolognesi PR. Manual prático de saúde e segurança no trabalho. São Caetano do Sul: Yendis; 2009

9. Eickmann UI, Falcy M, Fokuhl L, Rüegger M, Bloch M, Merz B. Surgical smoke: risks and preventive measures [Internet]. Hamburgo: International Social Security Association; 2012 [acessado em 10 mar. 2018]. Disponível em: http://prevencion.umh.es/files/2012/04/2-surgical_smoke.pdf

10. Barrett WL, Garber SM. Surgical smoke: a review of the literature. Surg Endosc. 2003;17(6):979-87. https://doi.org/10.1007/s00464-002-8584-5

11. Yeh CR. Surgical smoke plume: principles and function of smoke, aerosol, gases and smoke evacuators. Surg Serv Manag. 1997;3(4):41-5.

12. Boorder T, Verdaasdonk J, Klaessens J. The visualisation of surgical smoke produced by energy delivery devices: significance and effectiveness of evacuation systems. Proc of SPIE [Internet]. 2007 [acessado em 15 jul. 2017];6440;64400R-1. Disponível em: http:// www.buffalofilter.com/files/4614/1409/4386/The_visualisation_of_ surgical_smoke_produced_by_energy_delivery.pdf

13. Alp E, Bijlc D, Bleichrodt RP, Hansson B, Voss A. Surgical smoke and infection control. J Hosp Infect. 2006;62(1):1-5. https://doi. org/10.1016/j.jhin.2005.01.014

14. Tseng HS, Liu SP, Uang SN, Yang LR, Lee SC, Liu YJ, et al. Cancer risk of incremental exposure to polycyclic aromatic hydrocarbons in electrocautery smoke for mastectomy personnel. World J Surg Oncol. 2014;12:31. https://dx.doi.org/10.1186\%2F1477-7819-12-31

15. Claudio CV, Ribeiro RP, Martins JT, Marziale MHP, Solci MCD, Dalmas JC. Hidrocarbonetos policíclicos aromáticos produzidos pela fumaça do eletrocautério e uso de equipamentos de proteção individual. Rev Latino-Am Enferm. 2017;25:e2853. https://dx.doi. org/10.1590/1518-8345.1561.2853
16. Choi SH, Kwon TG, Chung SK, Kim TH. Surgical smoke may be a biohazard to surgeons performing laparoscopic surgery. Surg Endosc. 2014;28(8):2374-80. https://doi.org/10.1007/ s00464-014-3472-3

17. Wu YC, Tang CS, Huang HY, Liu CH, Chen YL, Chen DR, et al. Chemical production in electrocautery smoke by a novel predictive model. Eur Surg Res. 2011;46:102-7. http://dx.doi.org/10.1159/000322855

18. Tomita Y, Mihashi S, Nagata K, Ueda S, Fujiki M, Hirano M, et al. Mutagenicity of smoke condensates induced by $\mathrm{CO}$-laser irradiation and electrocauterization. Mutat Res. 1981;89(2):145-9.

19. Agency for Toxic Substances and Disease Registry. Polycyclic Aromatic Hydrocarbons (PAHs) [Internet]. 2011 [acessado em 28 fev. 2018]. Disponivel em: http://www.atsdr.cdc.gov/substances/toxsubstance. asp?toxid $=25$

20. Maquet Getinge Group. Smoke evacuation unit: MEDAP-Fumovac 900. Surgical smoke endangers the OR team [Internet]. 2017 [acessado em 20 jan. 2018]. Disponível em: http://online.tolmed.info/media/ brochures/fumovac_brochure_en-5_nonus.pdf

21. Benson SM, Novak DA, Ogg MJ. Proper use of surgical N95 respirators and surgical masks in the OR. AORN J. 2013;97(4):457-67. https:// doi.org/10.1016/j.aorn.2013.01.015

22. Institute for Safe Medication Practices. ISMP's list of error-prone abbreviations, symbols, and dose designations [Internet]. 2015 [acessado em 15 nov. 2017]. Disponível em: https://www.ismp.org/ tools/errorproneabbreviations.pdf

23. Association of periOperative Registered Nurses (AORN). RP Summary: recommended practices for electrosurgery. AORN J. 2012;95(3):388-90.

24. Ball K. Update for nurse anesthetists. Part 1. The hazards of surgical smoke. AANA J. 2001;69(2):125-32.

25. Lee KW, Mukund R. Filter collection. In: Baron PA, Welleke K, eds. Aerosol measurement, principles, techniques and applications. Nova York: Wiley Interscience; 2001. p.197-229.

26. Schultz L. An analysis of surgical smoke plume components, capture and evacuation. AORN J. 2014;99(2):289-98. https://doi.org/10.1016/j. aorn.2013.07.020 